Review Article

\title{
Associations between Gestational Diabetes and Anxiety or Depression: A Systematic Review
}

\author{
Hong OuYang, ${ }^{1}$ Bo Chen, ${ }^{2}$ Al-Mureish Abdulrahman $\mathbb{D}^{1},{ }^{1}$ Ling Li, ${ }^{1}$ and $\mathrm{Na} \mathrm{Wu} \mathbb{D}^{1,3}$ \\ ${ }^{1}$ Department of Endocrinology, Shengjing Hospital of China Medical University, Shenyang, China \\ ${ }^{2}$ Department of Endocrinology, The First People's Hospital of Kerqin District, Tongliao City, Inner Mongolia, China \\ ${ }^{3}$ Clinical Skills Practice Teaching Center, Shengjing Hospital of China Medical University, Shenyang, China
}

Correspondence should be addressed to $\mathrm{NaWu}$; 3441535223@qq.com

Hong OuYang and Bo Chen contributed equally to this work.

Received 31 March 2021; Revised 2 July 2021; Accepted 7 July 2021; Published 28 July 2021

Academic Editor: Peter Kempler

Copyright ( 92021 Hong OuYang et al. This is an open access article distributed under the Creative Commons Attribution License, which permits unrestricted use, distribution, and reproduction in any medium, provided the original work is properly cited.

Gestational diabetes mellitus (GDM) pregnant women are under more psychological stress than normal pregnant women. With the deepening of the study of gestational diabetes mellitus, research has shown that anxiety and depression are also an important cause of gestational diabetes mellitus. Anxiety and depression can cause imbalances in the hormone levels in the body, which has a serious impact on the pregnancy outcome and blood glucose control of pregnant women with GDM. Therefore, the main purpose of this paper is to provide a systematic review of the association between anxiety, depression, and GDM, as well as the adverse effects on pregnant women with GDM. To this end, we searched the PubMed, CNKI, Embase, Cochrane Library, Wanfang, and Weipu databases. Studies on the incidence of anxiety, depression, and GDM, blood glucose in pregnant women with GDM, delivery mode, and maternal and infant outcomes were included to be analyzed, and the source of anxiety and depression in pregnant women with GDM and related treatment measures were discussed.

\section{Introduction}

Gestational diabetes mellitus (GDM) is defined as carbohydrate intolerance resulting in hyperglycemia with first onset or detection during pregnancy [1], accounting for $86 \%$ of hyperglycemia during pregnancy [2]. Compared to healthy pregnant women, pregnant women with GDM are more likely to develop maternal and infant complications and are more likely to develop type 2 diabetes [3], cardiovascular disease, dyslipidemia, and metabolic disorders after delivery [4-6].

The mental health problems of pregnant women, especially the mental state of GDM, a high-risk group, have attracted a great attention from scholars all over the world. Studies in this population show that apart from physiological factors, anxiety and depression are also important causes of gestational diabetes [7]. However, there is no unified conclusion regarding the correlation between anxiety and depression and GDM. On the one hand, the study found that anxiety and depression can lead to chronic hypothalamicpituitary-adrenal hyperactivity, resulting in increased release of cortisol and insulin resistance [8], increasing risk of developing GDM in pregnant women. At the same time, the diagnosis of GDM may increase the risk of antenatal or postnatal depression through a reverse mechanism [9]. This suggests that there may be a two-way relationship between gestational diabetes and anxiety and depression. However, on the other hand, some studies believe that anxiety and depression do not increase the incidence of GDM in pregnant women [10-13], and the diagnosis of GDM does not increase the risk of prenatal or postnatal depression [14-16]. There is currently no consensus on the relationship between anxiety and depression and GDM. We therefore conducted a systematic review of the relevant literature to further explore the bidirectional relationship between anxiety and depression and GDM. At the same time, we further studied the effects of anxiety and depression on blood glucose and maternal and infant outcomes in pregnant women with GDM and 
discussed the sources of anxiety and depression and related treatment measures.

\section{Materials and Methods}

Using a computer, we retrieved studies from the PubMed, Chinese Journal Full Text (CNKI), EMBASE, Cochrane Library, Wan Fan, and Wei Pu databases. The retrieval period was from the inception of the databases to March 2021, using a combination of theme words and free words. Search terms included "gestational diabetes mellitus," "anxiety," "depression," "incidence of gestational diabetes mellitus," "blood glucose," "delivery mode," "maternal and infant outcomes," "influencing factors," and "treatment measures." There are no specific restrictions on the type, language, or location of the publication type of each study. Articles not directly relevant to the subject were excluded. The EndNote software was used to handle the proper references.

\section{Results and Discussion}

Following a predesigned literature retrieval strategy, 283 articles were retrieved, including 43 repeated articles. After reviewing the titles and briefs, another 175 were excluded, thus reviewing 65 articles in detail. Finally, we included 44 studies for discussion. The remaining 21 studies were excluded because they were not consistent with our research content. The literature screening process and the results are shown in Figure 1.

\subsection{Correlation between Anxiety, Depression, and GDM}

3.1.1. GDM Is an Associated Factor for Depression or Anxiety. Whether pregnant women with GDM experience higher levels of anxiety or depression than nonpregnant women has not been consistent in some previous studies. On the one hand, research suggests that pregnant women with GDM have been reported to experience higher levels of anxiety and stress than nonpregnant women and healthy pregnant women [17]. Diagnosis of GDM in pregnant women will increase their susceptibility to depression or anxiety $[18,19]$, resulting in $2-4$ times more likely to have antenatal or postnatal depression than women without GDM during pregnancy and after childbirth [20]. According to Egan et al. [21], compared with pregnant women with type 1 diabetes, pregnant women with GDM, and pregnant women without diabetes, it was found that pregnant women with diabetes were more prone to anxiety and depression than pregnant women without diabetes, among whom women with GDM had the highest scores of depression, anxiety, and stress. In a retrospective cohort study [22], Pace et al. found that pregnant women with GDM were twice as likely to be diagnosed with prenatal depression as those without GDM. However, on the other hand, in a large populationbased cohort study, GDM was found not to be associated with an increased risk of new-onset mental illness during pregnancy or postpartum [23]. This finding is consistent with the research done by Walmer et al. [24]. For that reason, aiming at existing contradictions, in 2019, Azami et al. con-

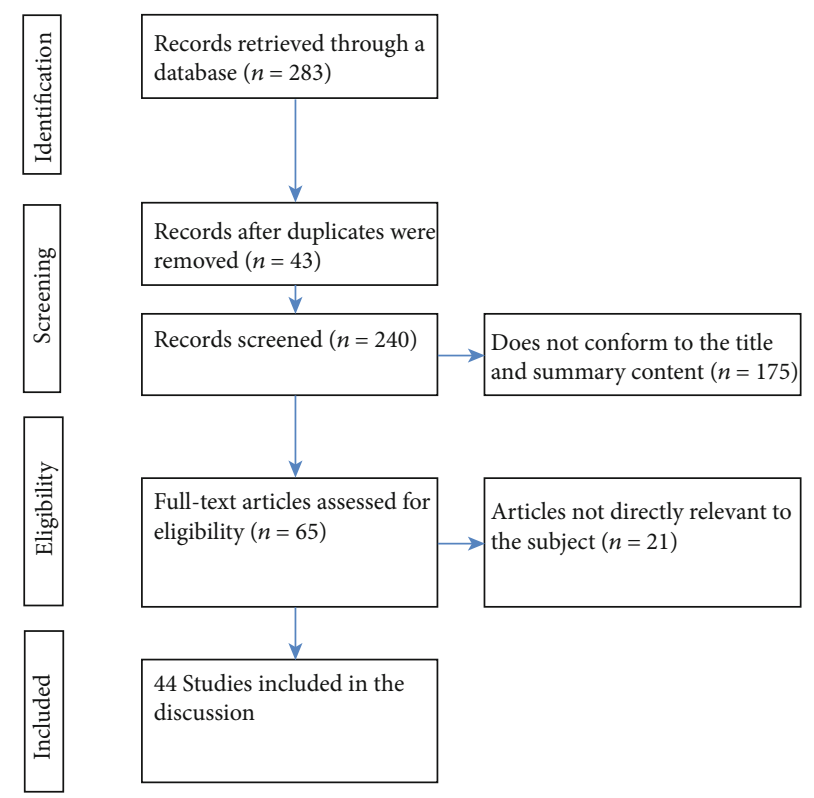

Figure 1: Flow diagram of studies identified.

ducted the first systematic review and meta-analysis of the relationship between gestational diabetes mellitus and postpartum depression [9]. This meta-analysis included 18 studies, and the final results showed that gestational diabetes significantly increased the risk of postpartum depression [9]. Based on the results of the meta-analysis by Milad, we currently believe that pregnant women with gestational diabetes are more prone to anxiety or depression. However, there is limited research on the relationship between gestational diabetes and postpartum depression, and more research is needed to confirm this.

3.1.2. Relationship between Anxiety and Depression and the Incidence of GDM. In recent years, anxiety and depression among pregnant women are very common [25]. Studies have suggested that anxiety and depression may be a risk factor for the occurrence of GDM $[26,27]$.

(1) Anxiety Is Associated with GDM. Bowers et al. believed that anxiety was a risk factor for the occurrence of GDM [28]; anxiety symptoms were found to significantly increase the risk of developing diabetes [29]. At the same time, Tang et al. conducted a prospective cohort study [30], and the results also showed that the incidence of GDM in pregnant women with anxiety symptoms was higher than that in pregnant women without anxiety symptoms.

(2) Depression Is Associated with GDM. The relationship between depression in early pregnancy and GDM is controversial. Tang et al. [30] explored the relationship between depression in early pregnancy (8-14 weeks) and GDM, but did not find that depression in early pregnancy is related to the occurrence of GDM. This is consistent with the results of Katon et al. and Byrn, who believed that the symptoms of depression did not increase the risk of GDM during the first trimester of pregnancy [31, 32]. However, in 2019, a 
relevant study has found [33] that depressed women are more likely to develop GDM in the first trimester of pregnancy than those who are not depressed. After controlling demographic factors and weight-related variables, depression in the first trimester was still predictive of future GDM development, and depression in the first trimester may indicate an increased risk of subsequent GDM. However, since there is no unified index for the definition of early pregnancy and the diagnosis of depression in various studies at present and the lack of relevant clinical control data, further studies are needed to prove whether depression in early pregnancy will increase the incidence of GDM.

3.2. Adverse Outcomes for Any Combination between GDM and Depression or Anxiety. Anxiety, depression, and other negative emotional states can cause imbalances in the body's hormone levels and increase blood sugar, which will have adverse effects on pregnant women with GDM $[34,35]$. We summarized the relevant studies (Table 1) to further discuss the effects of anxiety and depression on blood glucose, delivery mode, and maternal and infant outcomes in pregnant women with GDM [21, 33, 36-42].

3.2.1. Influence on Blood Glucose in Pregnant Women with $G D M$. Studies have suggested that anxiety and depression can cause excitement in the sympathetic adrenal medulla system; promote the secretion of ACTH; increase the levels of glucocorticoid, glucagon, and catecholamine; and finally accelerate the decomposition of gluconeogenesis and glycogen and increase the blood glucose levels of pregnant women [43]. In an earlier randomized controlled study, Wen et al. [39] found that the blood glucose of GDM pregnant women in the anxiety group was higher than that of the nonanxiety group and the blood glucose of GDM pregnant women in the depression group was higher than that of the nondepression group. The results [39] showed that pregnant women with negative emotions tended to have higher blood sugar, which was more difficult to control and those with depression were less optimistic about blood sugar levels. This is consistent with the results of later studies by Horsch et al. [27]. In addition, related studies also found that $\mathrm{HbAlc}$ in pregnant women with GDM in the anxiety group was significantly higher than that in the control group $[44,45]$ and the level of $\mathrm{HbAlc}$ was positively correlated with anxiety and depression scores in pregnant women with GDM [27]. It is worth noting that some studies $[31,46,47]$ suggest that different levels and types of anxiety were closely related to the HbAlc level. These studies divided GDM pregnant women into a high trait anxiety group and a low trait anxiety group according to the trait anxiety subscale in STAI (used to show the anxiety of personality trait type of patients) and found that the HbAlc of the high trait anxiety group was higher than that of the low trait anxiety group. The mechanism may be that patients with high trait anxiety have a stronger and more persistent response to stressful life events in their daily lives and are more likely to be in a state of high stress [48]. In general, combined with the current relevant studies, we believe that anxiety or depression during pregnancy has adverse effects on blood glucose and HbAlc levels in preg- nant women with GDM, and more clinical studies are needed in the future to provide data to further confirm this view.

\subsubsection{Influence on Delivery Mode of GDM Pregnant Women.}

The study found that, compared with the normal group of GDM pregnant women, the anxiety group of GDM pregnant women and the depression group of GDM pregnant women had higher cesarean section and forceps delivery rates, while the vaginal delivery rate was lower $[36,37,40]$. At the same time, the degree of depression in GDM pregnant women was more severe and the rate of vaginal delivery was lower, while the rate of cesarean section and prenatal assisted delivery was higher [40]. The reason why GDM pregnant women in the anxiety and depression group have bad delivery mode may be that anxiety and depression may lead to constant changes in neuroendocrine and intensify the pain of childbirth. At the same time, severe pain response may cause the coordination of uterine contractions to be out of balance, increasing the rate of cesarean section [36, 49]. However, these studies did not mention whether the prepregnancy BMI variable was controlled in pregnant women with GDM. One study [30] believes that there is a positive correlation between BMI and anxiety and depression in pregnant women before pregnancy, suggesting that with the increase of BMI, the incidence of anxiety and depression in pregnant women increases and the incidence of poor delivery methods increases. Therefore, regarding the influence of anxiety and depression on the delivery mode of pregnant women with GDM, we still need more studies to further exclude the role of relevant influencing factors.

3.2.3. Influence on Maternal and Infant Outcomes in Pregnant Women with GDM. Anxiety during pregnancy is a risk factor that leads to adverse outcomes for mothers and infants [10, 50-52]. Pregnant women with GDM experience prenatal depressive symptoms during pregnancy, which may be associated with an increase in adverse pregnancy outcomes [28, 53-55]. As early as 2000, Kurki et al. first reported an association between prenatal depression and an increased incidence of adverse perinatal outcomes in pregnant women [56]. In recent years, a number of studies have found that compared with the normal GDM group, GDM pregnant women in the anxiety and depression group had significantly higher incidences of adverse maternal and infant outcomes, including significantly lower neonatal weight, premature rupture of membranes, postpartum hemorrhage, postpartum infection, macrosomia, neonatal asphyxia, and neonatal hypoglycemia [36, 37, 40]. And there was a positive correlation between the degree of depression and the adverse maternal and infant outcomes in pregnant women with GDM [40]. Such as in 2019, Packer et al. [41] found that women with GDM and depression had significantly higher rates of preeclampsia, gestational hypertension, and preterm birth. The reason may be that depression is associated with vascular changes that may increase the risk of preeclampsia and hypertension [56, 57]. In 2020, Lee et al. [58] found in a cross-sectional study of pregnant women with gestational diabetes in Malaysia that depression, anxiety, or stress in pregnant women with GDM during pregnancy may increase 


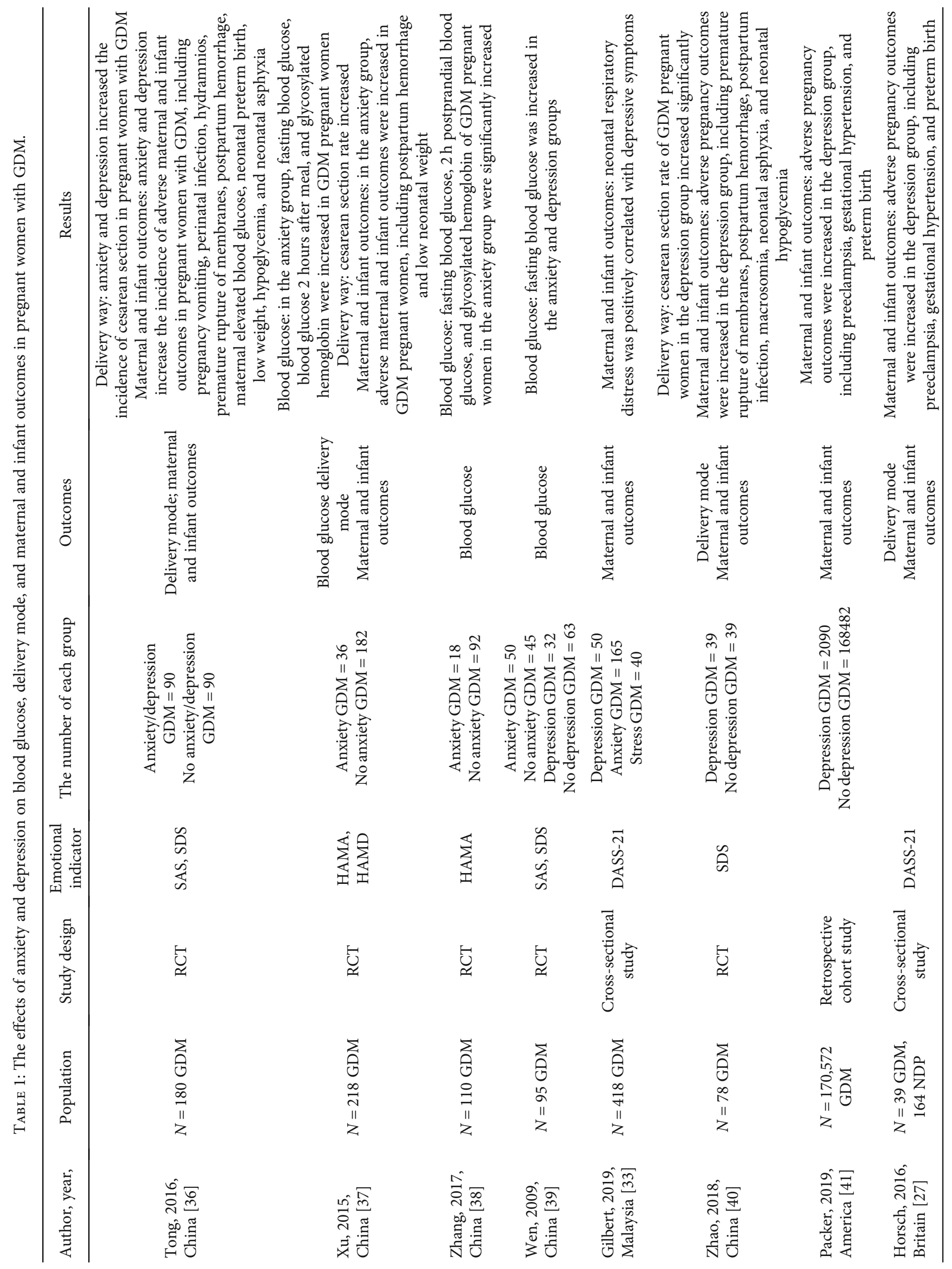




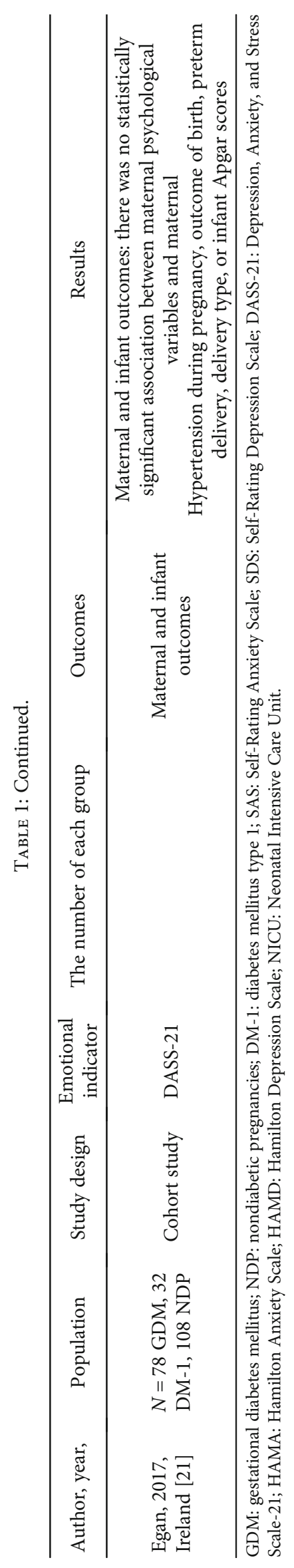


the risk of neonatal morbidity and mortality, eventually leading to adverse neonatal outcomes. In the future, we need to conduct more studies to further explore the relationship between adverse emotions and maternal and infant outcomes.

3.3. Related Influencing Factors of Anxiety and Depression in Pregnant Women with GDM. The study on the relevant influencing factors of anxiety and depression in pregnant women with GDM will help us to better understand the source of these negative emotions, provide guidance on intervention measures, improve the prenatal mood of pregnant women, and reduce the impact of anxiety and depression on pregnancy outcomes.

3.3.1. The Influence of Gestational Diabetes Diagnosis on Pregnancy Complicated with Anxiety and Depression. Studies have found that women with GDM are more prone to prenatal depression, anxiety, and stress when they are aware that uncontrolled diabetes can lead to pregnancy-related complications and adverse neonatal outcomes $[59,60]$. Once pregnant women know that they have gestational diabetes, one of the sources of anxiety and depression may be that the patient is unable to face their illness correctly and has serious concerns about the disease, which causes certain psychological pressure. Studies have found that the cognition of gestational diabetes among patients is closely related to the occurrence of psychological problems [48, 61]; that is, due to the lack of related professional knowledge of diabetes, the knowledge of diabetes treatment, and the common sense of daily nursing, patients are more worried about the disease, which is more likely to produce anxiety and depression. Another source may come from the need for selfmonitoring of blood glucose, dietary restrictions, and the need for insulin treatment [62]. Patients are afraid of treatment and are concerned about the adverse effects of medication on the growth and development of the fetus, resulting in anxiety $[15,48,63]$. Through research [16], Hui et al. divided the sources of anxiety and depression in pregnant women with GDM into three themes: Theme 1 -stress related to diagnosis of GDM and perception of high-risk pregnancy; Theme 2-stress associated with losing control of GDM during the dietary management process; and Theme 3-anxiety related to fear of maternal and infant complications. Among them, the study identified concerns about maternal and infant complications (Theme 3) as the biggest source of stress for women with GDM. At the same time, according to this study [16], compared with women who only received dietary treatment, women who received insulin treatment experienced significantly higher levels of stress. This is consistent with the latest study by Lee et al., which found [64] that pregnant women with GDM who received insulin treatment were more likely to have symptoms of anxiety and depression, which may be due to a significant correlation between insulin and hypoglycemia episodes, thus exacerbating patients' concerns about treatment.

3.3.2. The Influence of Various Factors in Pregnant Women with Gestational Diabetes. Women with low family income
[65], low socioeconomic status [66], low educational level $[66,67]$, and high body mass index (BMI) [68] have been reported to have an increased rate of antenatal depression. At the same time, occupation, marital status of pregnant women, and negative life events during pregnancy (daily quarrel) were all related to pregnancy depression. Relevant studies $[68,69]$ showed that the age of pregnant women with GDM was positively correlated with the prevalence of anxiety and depression, suggesting that the incidence of anxiety and depression increased with age. At the same time, the study found that adverse birth history was positively correlated with anxiety and depression in pregnant women, suggesting that GDM pregnant women with adverse birth history are prone to anxiety and depression $[68,69]$. This is consistent with the findings of Tsartsara and Johnson [70]. In addition, Lee et al. [64] found that a family history of depression and anxiety is an important factor in the development of depression and anxiety symptoms, which may be due to the fact that people with a family history of depression and anxiety inherit the genes for psychiatric disorders and may manifest themselves when they are under stress, especially after the diagnosis of GDM. A history of depression was also found to be significantly associated with an increased risk of GDM in a large multiethnic cohort study in the United States [28].

3.4. Treatment Measures. In the above studies, we found that pregnant women with gestational diabetes had a higher incidence of anxiety and depression symptoms, suggesting that the mental health level of pregnant women with gestational diabetes was worse than that of normal pregnant women. Anxiety, depression, and other emotions not only affect the outcome of a pregnant woman's own diseases but also harm the growth of the fetus. At the same time, the aggravation of the disease and complications will also lead to or aggravate these bad emotions, forming a vicious circle. Therefore, in addition to the application of drug therapy, pregnant women with GDM also need medical staff to pay attention to the negative emotions of pregnant women with GDM, to carry out targeted psychological counseling for pregnant women, and to take more effective intervention measures as soon as possible to avoid the occurrence of adverse pregnancy outcomes and promote maternal and child health. Relevant studies have shown that comprehensive psychological intervention can help patients to understand gestational diabetes correctly, enhance patients' confidence, and actively cooperate with the treatment of medical staff $[15,48]$. Individualized health education can help patients understand the relevant professional knowledge of diabetes, so that patients can see gestational diabetes correctly and thus improve their confidence in treatment $[71,72]$. Supportive measures, including spiritual comfort and psychological support, can not only create a healthy and pleasant therapeutic atmosphere to help patients reduce psychological stress, improve cognitive level, and eliminate psychosomatic and psychosomatic reactions but can also help medical staff to understand the patient's personality characteristics to develop appropriate psychological and behavioral treatment measures and procedures. At present, for the treatment of pregnant women with GDM, some organizations suggest starting with diet and exercise 
therapy to try to achieve a normal blood glucose level [7375]. Therefore, for pregnant women with GDM who have anxiety and depression, a healthy lifestyle is also essential, including a reasonable diet and appropriate exercise. On the one hand, strict diabetic diet control and appropriate exercise can help patients better control blood glucose during pregnancy $[76,77]$, and good blood glucose control can also enhance patients' enthusiasm for treatment and ease their anxiety. On the other hand, studies have found that diet and exercise can also reduce the occurrence of adverse pregnancy outcomes in pregnant women with GDM [78], so it can also reduce the adverse effects of anxiety and depression to a certain extent.

\section{Conclusions}

In the above study, we found that anxiety or depression during pregnancy increased the incidence of GDM in pregnant women to a certain extent. And the diagnosis of GDM will increase the incidence of anxiety and depression in pregnant women. At the same time, our study also found that compared with normal GDM pregnant women, GDM pregnant women with anxiety and depression were more likely to have adverse outcomes in terms of blood glucose during pregnancy, delivery mode, and maternal and infant outcomes. Further analysis of the source of anxiety and depression in pregnant women with GDM shows that the diagnosis of gestational diabetes and various factors affecting pregnant women with GDM themselves play a role. As a result, with the increase in the incidence of GDM, the psychological problems of GDM patients also deserve attention. Active and effective psychological intervention measures should be taken to help GDM pregnant women pass the pregnancy period safely and achieve better pregnancy outcomes.

\section{Consent}

All authors agreed to publish.

\section{Disclosure}

The authors declare no conflicts of interest in this work.

\section{Conflicts of Interest}

None is declared.

\section{Authors' Contributions}

Hong OuYang and Bo Chen devised the project, collected the material, and wrote the manuscript. Al-Mureish Abdulrahman polished the text. $\mathrm{Na} \mathrm{Wu}$ and Ling Li helped with the discussion, supervised the project, and provided critical feedback. All authors discussed and contributed to the final manuscript. And all authors have read and approved the manuscript. Hong Ouyang and Bo Chen are the co-first authors.

\section{Acknowledgments}

This research was supported by the National Natural Science Foundation of China (No. 81700706), the 345 Talent Project of Shengjing Hospital, the Clinical Research Project of Liaoning Diabetes Medical Nutrition Prevention Society (No. LNSTNBYXYYFZXH-RS01B), the Science Foundation of Education Department of Liaoning Province (No. LK201603), and the Virtual Simulation Experiment Teaching Project of China Medical University (No. 2020-47). We gratefully acknowledge Shengwei Yu for providing intellectual support and technical assistance.

\section{References}

[1] C. Gao, X. Sun, L. Lu, F. Liu, and J. Yuan, "Prevalence of gestational diabetes mellitus in mainland China: a systematic review and meta-analysis," J Diabetes Investig, vol. 10, no. 1, pp. 154-162, 2019.

[2] N. H. Cho, J. E. Shaw, S. Karuranga et al., "IDF diabetes atlas: global estimates of diabetes prevalence for 2017 and projections for 2045," Diabetes Research and Clinical Practice, vol. 138, pp. 271-281, 2018.

[3] E. Vounzoulaki, K. Khunti, S. C. Abner, B. K. Tan, M. J. Davies, and C. L. Gillies, "Progression to type 2 diabetes in women with a known history of gestational diabetes: systematic review and meta-analysis," BMJ, vol. 369, 2020.

[4] B. Akinci, A. Celtik, S. Genc et al., "Evaluation of postpartum carbohydrate intolerance and cardiovascular risk factors in women with gestational diabetes," Gynecological endocrinology: the official journal of the International Society of Gynecological Endocrinology, vol. 27, no. 5, pp. 361-367, 2011.

[5] B. Akinci, A. Celtik, S. Yener, and S. Yesil, "Prediction of developing metabolic syndrome after gestational diabetes mellitus," Fertility and Sterility, vol. 93, no. 4, pp. 1248-1254, 2010.

[6] B. Akinci, A. Celtik, F. Yuksel et al., "Increased osteoprotegerin levels in women with previous gestational diabetes developing metabolic syndrome," Diabetes Research and Clinical Practice, vol. 91, no. 1, pp. 26-31, 2011.

[7] L. Mei, L. Jian, and C. Yongyi, "Relationship between life events and mental health status of pregnant women with gestational diabetes mellitus in Guangzhou," China Journal of Health Psychology, vol. 25, no. 3, pp. 336-339, 2017.

[8] D. J. Robinson, M. Coons, H. Haensel, M. Vallis, and J. F. Yale, "Diabetes and mental health," Canadian Journal of Diabetes, vol. 42, pp. S130-S141, 2018.

[9] M. Azami, G. Badfar, A. Soleymani, and S. Rahmati, "The association between gestational diabetes and postpartum depression: a systematic review and meta-analysis," Diabetes Research and Clinical Practice, vol. 149, pp. 147-155, 2019.

[10] J. Martini, S. Knappe, K. Beesdo-Baum, R. Lieb, and H. U. Wittchen, "Anxiety disorders before birth and self-perceived distress during pregnancy: associations with maternal depression and obstetric, neonatal and early childhood outcomes," Early Human Development, vol. 86, no. 5, pp. 305-310, 2010.

[11] H. Bayrampour, C. Salmon, A. Vinturache, and S. Tough, "Effect of depressive and anxiety symptoms during pregnancy on risk of obstetric interventions," The Journal of Obstetrics and Gynaecology Research, vol. 41, no. 7, pp. 1040-1048, 2015.

[12] U. Ghimire, S. Papabathini, J. Kawuki, N. Obore, and T. H. Musa, "Depression during pregnancy and the risk of low birth 
weight, preterm birth and intrauterine growth restriction- an updated meta-analysis," Early Human Development, vol. 152, article 105243, 2021.

[13] J. Dayan, C. Creveuil, M. N. Marks et al., "Prenatal depression, prenatal anxiety, and spontaneous preterm birth: a prospective cohort study among women with early and regular care," Psychosomatic Medicine, vol. 68, no. 6, pp. 938-946, 2006.

[14] A. Arafa and J. Dong, "Depression and risk of gestational diabetes: a meta-analysis of cohort studies," Diabetes Research and Clinical Practice, vol. 156, article 107826, 2019.

[15] N. B and L. Yi, "Investigation on mental health status of gestational diabetes mellitus patients," International Journal of Nursing, vol. 37, no. 2, pp. 167-172, 2018.

[16] A. Hui, G. Sevenhuysen, D. Harvey, and E. Salamon, "Stress and anxiety in women with gestational diabetes during dietary management," The Diabetes Educator, vol. 40, no. 5, pp. 668677, 2014.

[17] M. Hayase, M. Shimada, and H. Seki, "Sleep quality and stress in women with pregnancy-induced hypertension and gestational diabetes mellitus," Women and birth : journal of the Australian College of Midwives, vol. 27, no. 3, pp. 190-195, 2014.

[18] K. Hjelm, K. Berntorp, A. Frid, A. Åberg, and J. Apelqvist, "Beliefs about health and illness in women managed for gestational diabetes in two organisations," Midwifery, vol. 24, no. 2, pp. 168-182, 2008.

[19] J. Hirst, T. Tran, M. A. T. Do, F. Rowena, J. M. Morris, and H. E. Jeffery, "Women with gestational diabetes in Vietnam: a qualitative study to determine attitudes and health behaviours," BMC Pregnancy and Childbirth, vol. 12, no. 1, 2012.

[20] S. Hinkle, G. Buck Louis, S. Rawal, Y. Zhu, P. S. Albert, and C. Zhang, "A longitudinal study of depression and gestational diabetes in pregnancy and the postpartum period," Diabetologia, vol. 59, no. 12, pp. 2594-2602, 2016.

[21] A. M. Egan, F. P. Dunne, K. Lydon, S. Conneely, K. Sarma, and B. E. McGuire, "Diabetes in pregnancy: worse medical outcomes in type 1 diabetes but worse psychological outcomes in gestational diabetes," QJM : Monthly Journal of the Association of Physicians, vol. 110, no. 11, pp. 721-727, 2017.

[22] R. Pace, E. Rahme, D. da Costa, and K. Dasgupta, “Association between gestational diabetes mellitus and depression in parents: a retrospective cohort study," Clinical Epidemiology, vol. Volume 10, pp. 1827-1838, 2018.

[23] Q. Beka, S. Bowker, A. Savu, D. Kingston, J. A. Johnson, and P. Kaul, "Development of perinatal mental illness in women with gestational diabetes mellitus: a population-based cohort study," Canadian Journal of Diabetes, vol. 42, no. 4, pp. 350355.e1, 2018.

[24] R. Walmer, J. Huynh, J. Wenger et al., "Mental health disorders subsequent to gestational diabetes mellitus differ by race/ethnicity," Depression and Anxiety, vol. 32, no. 10, pp. 774-782, 2015.

[25] X. Ma, "Study on anxiety and depression level and its influencing factors in pregnant women Exhibition," China Maternal and Child Health, vol. 34, no. 3, pp. 722-724, 2019.

[26] M. Byrn and S. Penckofer, "The relationship between gestational diabetes and antenatal depression," Journal of Obstetric, Gynecologic, and Neonatal Nursing, vol. 44, no. 2, pp. 246-255, 2015.

[27] A. Horsch, J. S. Kang, Y. Vial et al., "Stress exposure and psychological stress responses are related to glucose concentrations during pregnancy," British Journal of Health Psychology, vol. 21, no. 3, pp. 712-729, 2016.
[28] K. Bowers, S. K. Laughon, S. Kim et al., "The association between a medical history of depression and gestational diabetes in a large multi-ethnic cohort in the United States," Paediatric and Perinatal Epidemiology, vol. 27, no. 4, pp. 323-328, 2013.

[29] S. S. Hasan, K. Thiruchelvam, S. I. Ahmed, A. M. Clavarino, A. A. Mamun, and T. Kairuz, "Pregnancy complications, mental health-related problems and type 2 diabetes mellitus in Malaysian women," Diabetes \& Metabolic Syndrome, vol. 7, no. 4, pp. 191-197, 2013.

[30] Y. Tang, X. Lan, Y. Zhang et al., "Anxiety and depression on gestational diabetes mellitus in early pregnancy," Journal of Hygiene Reserch, vol. 49, no. 2, pp. 179-184, 2020.

[31] J. G. Katon, J. Russo, A. R. Gavin, J. L. Melville, and W. J. Katon, "Diabetes and depression in pregnancy: is there an association?," Journal of Women's Health, vol. 20, no. 7, pp. 983-989, 2011.

[32] S. Nehbandani, F. Nahidi, N. Kariman, and M. Nasiri, "Relationship between gestational diabetes and postpartum depression," Journal of Obstetric, Gynecologic, \& Neonatal Nursing, vol. 19, no. 7, pp. 18-24, 2015.

[33] L. Gilbert, J. Gross, S. Lanzi, D. Y. Quansah, J. Puder, and A. Horsch, "How diet, physical activity and psychosocial well-being interact in women with gestational diabetes mellitus: an integrative review," BMC Pregnancy and Childbirth, vol. 19 , no. 1, p. 60, 2019.

[34] H. Gao, T. Geng, T. Huang, and Q. Zhao, "Fish oil supplementation and insulin sensitivity: a systematic review and meta-analysis," Lipids in Health and Disease, vol. 16, no. 1, p. 131, 2017.

[35] J. L. L. Mei, L. Jian, C. Yongyi, and W. Chunwan, "Relationship between life events and mental health status of pregnant women with gestational diabetes in guangzhou," China Journal of Health Psychology, vol. 25, no. 3, pp. 336-339, 2017.

[36] D. Chengxian and L. Jin, "Effects of anxiety and depression on pregnancy outcome in pregnant women with gestational diabetes mellitus," Journal of International Psychiatry, vol. 43, no. 1, pp. 178-181, 2016.

[37] X. Yuanjiao and W. Zhijun, The influence of anxiety on gestational diabetes, Modern Hospital, 2015.

[38] Z. Yin and L. Junjie, "The effect of anxiety on blood glucose in pregnant women with gestational diabetes mellitus and the effect of psychological intervention combined with biofeedback training," China Journal of Health Psychology, vol. 25, no. 12, pp. 1784-1787, 2009.

[39] W. Zhi and R. Suqin, "Investigation and analysis of anxiety and depression in pregnant women with gestational diabetes mellitus," Chinese Journal of Modern Nursing, vol. 15, no. 20, pp. 1935-1937, 2009.

[40] Z. Lijuan, "Investigation of depression in patients with gestational diabetes mellitus and its relationship with delivery style and adverse pregnancy outcome," Journal of International Psychiatry, vol. 45, no. 1, pp. 144-146, 2018.

[41] C. H. Packer, R. A. Pilliod, L. R. Chatroux, A. B. Caughey, and A. M. Valent, "Increased rates of adverse perinatal outcomes in women with gestational diabetes and depression," The Journal of Maternal-Fetal \& Neonatal Medicine, vol. 32, pp. 1-5, 2019.

[42] C. Morrison, J. G. McCook, and B. A. Bailey, "First trimester depression scores predict development of gestational diabetes mellitus in pregnant rural Appalachian women," Journal of Psychosomatic Obstetrics and Gynaecology, vol. 37, no. 1, pp. 21-25, 2016. 
[43] Z. Xiaoming, "The characteristics of the dominant frequency of the a- wave and its influence on the psychological behavior in anxious adolescents," China Journal of Health Psychology, vol. 21, no. 7, pp. 989-999, 2013.

[44] M. Heaman, D. Kingston, M. Brownell, and M. Helewa, Perinatal services and outcomes in Manitoba, Manitoba Centre for Health Policy, Winnipeg, MB, USA, 2012.

[45] K. A. Ertel, M. Silveira, P. Pekow et al., "Prenatal depressive symptoms and abnormalities of glucose tolerance during pregnancy among Hispanic women," Archives of Women's Mental Health, vol. 17, no. 1, pp. 65-72, 2014.

[46] K. W. Lee, S. M. Ching, N. K. Devaraj et al., "Diabetes in pregnancy and risk of antepartum depression: a systematic review and meta-analysis of cohort studies," International Journal of Environmental Research and Public Health, vol. 17, no. 11, article 3767, 2020.

[47] K. W. Lee, H. C. Loh, S. C. Chong et al., "Prevalence of anxiety among gestational diabetes mellitus patients: a systematic review and meta-analysis," World Journal of Meta-Analysis, vol. 8, no. 3, pp. 275-284, 2020.

[48] Y. Baorong, "Clinical investigation and analysis of mental health status of pregnant women with gestational diabetes mellitus," Chinese Journal of Modern Drug Application, vol. 9, no. 22, pp. 242-244, 2015.

[49] L. Xiaofeng, W. Fang, H. Guiying, F. Xiaodong, and M. Xiguang, "The relationship between anxiety and depression during pregnancy and pregnancy outcome in women undergoing in vitro fertilization-embryo transfer," Journal of Practical Obstetrics and Gynecology, vol. 32, no. 6, pp. 456-459, 2016.

[50] C. Dunkel Schetter, "Psychological science on pregnancy: stress processes, biopsychosocial models, and emerging research Issues," Annual Review of Psychology, vol. 62, no. 1, pp. 531-558, 2011.

[51] P. Thiagayson, G. Krishnaswamy, M. L. Lim et al., "Depression and anxiety in Singaporean high-risk pregnancies - prevalence and screening," General Hospital Psychiatry, vol. 35, no. 2, pp. 112-116, 2013.

[52] T. Field, M. Diego, M. Hernandez-Reif et al., "Comorbid depression and anxiety effects on pregnancy and neonatal outcome," Infant Behavior \& Development, vol. 33, no. 1, pp. 2329, 2010.

[53] C. Larsson, G. Sydsjö, and A. Josefsson, "Health, sociodemographic data, and pregnancy outcome in women with antepartum depressive symptoms," Obstetrics and Gynecology, vol. 104, no. 3, pp. 459-466, 2004.

[54] A. Ruohomäki, E. Toffol, S. Upadhyaya et al., "The association between gestational diabetes mellitus and postpartum depressive symptomatology: a prospective cohort study," Journal of Affective Disorders, vol. 241, pp. 263-268, 2018.

[55] R. Hu, Y. Li, Z. Zhang, and W. Yan, “Antenatal depressive symptoms and the risk of preeclampsia or operative deliveries: a meta-analysis," PLoS One, vol. 10, no. 3, article e0119018, 2015.

[56] T. Kurki, V. Hiilesmaa, R. Raitasalo, H. Mattila, and O. Ylikorkala, "Depression and anxiety in early pregnancy and risk for preeclampsia," Obstetrics and Gynecology, vol. 95, no. 4, pp. 487-490, 2000.

[57] L. Meng, D. Chen, Y. Yang, Y. Zheng, and R. Hui, "Depression increases the risk of hypertension incidence," Journal of Hypertension, vol. 30, no. 5, pp. 842-851, 2012.

[58] K. Lee, S. Ching, F. K. Hoo et al., "Neonatal outcomes and its association among gestational diabetes mellitus with and with- out depression, anxiety and stress symptoms in Malaysia: a cross- sectional study," Midwifery, vol. 81, article 102586, 2020.

[59] C. Kim, P. Brawarsky, R. A. Jackson, E. Fuentes-Afflick, and J. S. Haas, "Changes in health status experienced by women with gestational diabetes and pregnancy-induced hypertensive disorders," Journal of Women's Health, vol. 14, no. 8, pp. 729736, 2005.

[60] M. Dalfrà, A. Nicolucci, T. Bisson, B. Bonsembiante, A. Lapolla, and QLISG (Quality of Life Italian Study Group), "Quality of life in pregnancy and post-partum: a study in diabetic patients," Quality of Life Research: an International Journal of Quality of Life Aspects of Treatment, Care and Rehabilitation, vol. 21, no. 2, pp. 291-298, 2012.

[61] N. Hongxia, Investigation on mental health status of gestational diabetes mellitus patients, Modern Chinese Doctor, 2011.

[62] T. Miazgowski, M. Bikowska, J. Ogonowski, and A. Taszarek, "The impact of health locus of control and anxiety on selfmonitored blood glucose concentration in women with gestational diabetes mellitus," Journal of Women's Health, vol. 27, no. 2, pp. 209-215, 2018.

[63] L. Haiying, C. Jingsi, and Z. Danxi, "Clinical investigation on mental health status of gestational diabetes patients," Maternal and Child Health Care of China, vol. 23, no. 27, pp. 3868-3870, 2008.

[64] K. W. Lee, S. M. Ching, F. K. Hoo et al., "Prevalence and factors associated with depressive, anxiety and stress symptoms among women with gestational diabetes mellitus in tertiary care centres in Malaysia: a cross-sectional study," BMC Pregnancy and Childbirth, vol. 19, no. 1, p. 367, 2019.

[65] S. Liqing, L. Jinlian, and T. Min, "Investigation of pregnant women's pregnancy stress and its influencing factors," Chinese Journal of Nursing, vol. 48, no. 9, pp. 808-811, 2013.

[66] R. H. Glazier, F. J. Elgar, V. Goel, and S. Holzapfel, "Stress, social support, and emotional distress in a community sample of pregnant women," Journal of Psychosomatic Obstetrics and Gynaecology, vol. 25, no. 3-4, pp. 247-255, 2004.

[67] C. Westdahl, S. Milan, U. Magriples, T. S. Kershaw, S. S. Rising, and J. R. Ickovics, "Social support and social conflict as predictors of prenatal depression," Obstetrics and Gynecology, vol. 110, no. 1, pp. 134-140, 2007.

[68] L. Zhiru and G. Xin, "Analysis of anxiety and depression related factors in patients with gestational diabetes mellitus," Chinese Journal of Medicine, vol. 48, no. 4, pp. 60-62, 2013.

[69] L. Shuiyu and K. Xiuquan, "Clinical investigation on mental health status of patients with gestational diabetes mellitus," Chinese Medical Innovations, vol. 16, no. 16, pp. 109-112, 2019.

[70] E. Tsartsara and M. Johnson, "The impact of miscarriage on women's pregnancy-specific anxiety and feelings of prenatal maternal-fetal attachment during the course of a subsequent pregnancy: an exploratory follow-up study," Journal of Psychosomatic Obstetrics and Gynaecology, vol. 27, no. 3, pp. 173182, 2006.

[71] S. Yonghui and X. Qian, "Investigation and analysis of mental health status of pregnant patients with diabetes mellitus," Maternal and Child Health Care of China, vol. 32, no. 9, pp. 1998-2001, 2017.

[72] S. Jiangchuan and C. Shufan, "Investigation on mental health status of pregnant women with diabetes mellitus," Journal of 
Chongqing Medical University, vol. 31, no. 1, pp. 126-128, 2006.

[73] IDF GDM Model of Care, Implementation Protocol: Guidelines for Healthcare Professionalshttps://www.idf.org/e-library/ guidelines/77-idf-gdm-model-of-care-implementationprotocol-guidelines-forhealthcareprofessionals.html.

[74] Diabetes in pregnancy: management from preconception to the postnatal period. NICE guidelineFebuary 2015, https://www .nice.org.uk/guidance/ng3.

[75] American Diabetes Association, "14. Management of diabetes in pregnancy: standards of medical care in diabetes-2019," Diabetes Care, vol. 42, Supplement 1, pp. S165-S172, 2019.

[76] P. J. Abernethy, R. Thayer, and A. W. Taylor, "Acute and chronic responses of skeletal muscle to endurance and sprint exercise. A review," Sports Medicine, vol. 10, no. 6, pp. 365389, 1990.

[77] T. E. Jensen and E. A. Richter, "Regulation of glucose and glycogen metabolism during and after exercise," The Journal of Physiology, vol. 590, no. 5, pp. 1069-1076, 2012.

[78] E. Shepherd, J. C. Gomersall, J. Tieu, S. Han, C. A. Crowther, and P. Middleton, "Combined diet and exercise interventions for preventing gestational diabetes mellitus," Cochrane Database of Systematic Reviews, vol. 11, 2017. 